\title{
The Communication Design of the Optical Fiber Reflective Memory Network in the Semi-physical Simulation System of the Laser Beam Rider Guidance Missile
}

\author{
YU Tianpeng ${ }^{1, a}$, CHEN Yongbo ${ }^{2, b}$, LI Jinlinag ${ }^{1, c}$ \\ ${ }^{1}$ Huaihai industrial group co., LTD, Norinco Group, Shanxi 046012, China \\ ${ }^{2}$ Beijing Institute of Technology, Beijing 100081, China \\ atianpeng_yu@sina.com, bbit_chenyongbo@163.com, jijinliang_li01@sina.com
}

Keywords: Optical fiber reflective memory network, semi-physical simulation system, laser beam rider guidance missile

\begin{abstract}
This paper introduces to set up an optical fiber reflective memory network, which needs the high transmission rate and the low data transmission delay in the data transmission process, in the semi-physical simulation system of the laser beam rider guidance missile because of the needs of the system. Practical application shows that all nodes of this system can transfer the interrupt, messages or data block to other nodes transparently and certainly. This network also can extend well beyond other simulation systems.
\end{abstract}

\section{Introduction}

The semi-physical simulation system of the laser beam rider guidance missile is to run a system which relates the mathematical model, the solid model and the physical device of system together. However, in the simulation process, because the real object is introduced into the simulation loop, the simulation needs to be completed in real time. The simulator receives the dynamic input signal and obtains the dynamic output response in real-time under the condition of synchronizing with the real system. The real-time ability is the premise of the semi-physical simulation system. Therefore, the data transmission delay must be low in the network data transmission process $[1,2]$.

The optical fiber reflective memory network is a real-time network system based on the internal storage. All these works are finished by the hardware without the software overheads. With the addition of the application of the optical fiber transmission medium, the data transmission rate and the data transmission delay can reach many tens of megabytes and several nanoseconds respectively. What's more, the data transmission delay of this system can be determined and predictable which is very difficult to achieve for the traditional network. It can support the interconnection of 256 nodes. At the same time, it supports different kinds of computers whose bus structures are PCI/VME/PMC, and it also can use the different operating systems to share the high-speed, steady-rate and real-time data. The internal storage of the optical fiber reflection (VMIC) has the advantages of the high bandwidth, the low latency and the lower cost.

The operating principle of the optical fiber reflective memory network is to insert the internal storage of the optical fiber reflection in every node which needs the real-time communication. In every card, it has its own independent local memory. It can map the independent local memory of the network card into the host memory by the local memory mapping. By this way, the users can read and write the data of the network cards quickly and conveniently as reading and writing upon the host memory. In addition, every optical fiber reflection internal storage card maps the local memory distributed on the node cards onto a virtual global memory by the network memory mapping. In other words, every node writes in the data of the local node card, meanwhile, it writes in the memory of the other node cards. Thus the read-write for the local node by the users is equivalent to the read-write for the global memory. The global memory is sharable for all distributed nodes. So it can realize the real-time data communication among the distributed nodes. By this way, all nodes can transfer the 
interrupt, messages or data block to other nodes transparently and certainly. The work rate reaches $2.1 \mathrm{G}$ baud rate. The next reflective memory card in the network receives this new data. Then the local memory will be updated in 400ns.

\section{The whole scheme design of the optical fiber reflective memory network for the real-time simulation}

Based on the characteristics and needs of the semi-physical simulation of the laser beam rider guidance missile, the whole system needs seven computer nodes to take part in the simulation. The simulation network system is composed of the ring topology group. These seven nodes respectively are the console computer, the Galaxy Simulation Workstation, the turntable control computer, the air source control computer, the movement servomechanism, the laser field simulator control computer, the visual display computer and the database computer [5]. The whole system is shown in Fig. 1 and Fig.2:

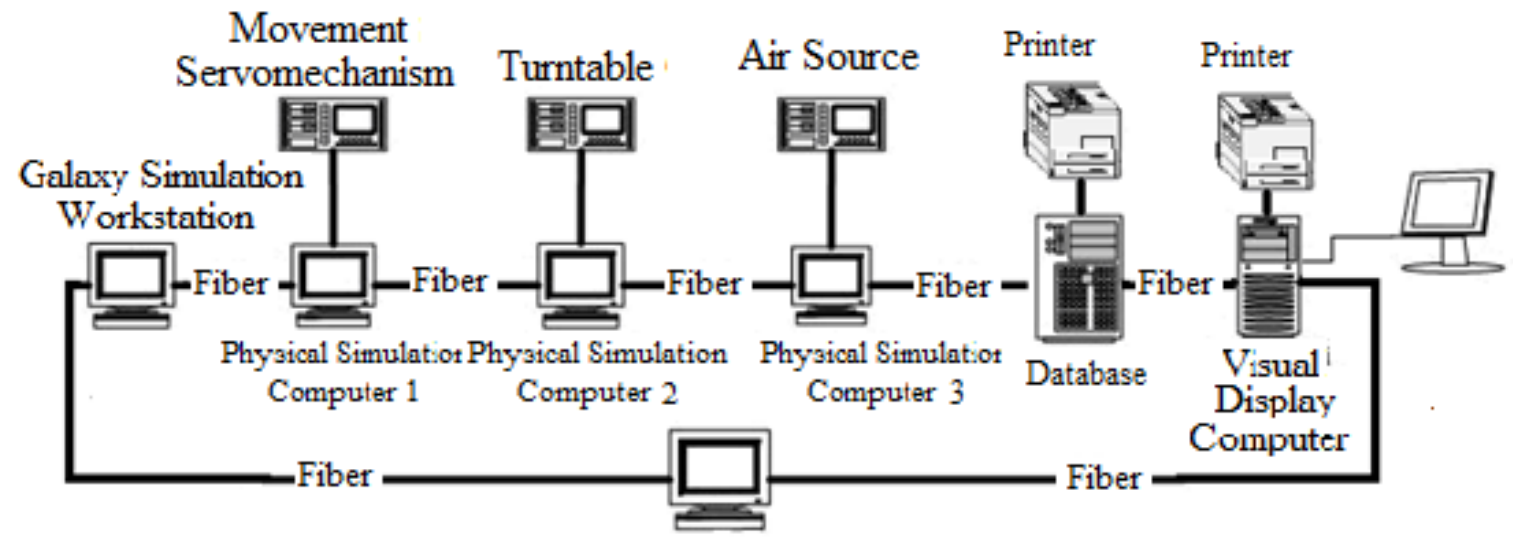

Console Computer

Fig. 1 The constitutional diagram of the Semi-physical Simulation System of the Laser Beam Rider Guidance Missile

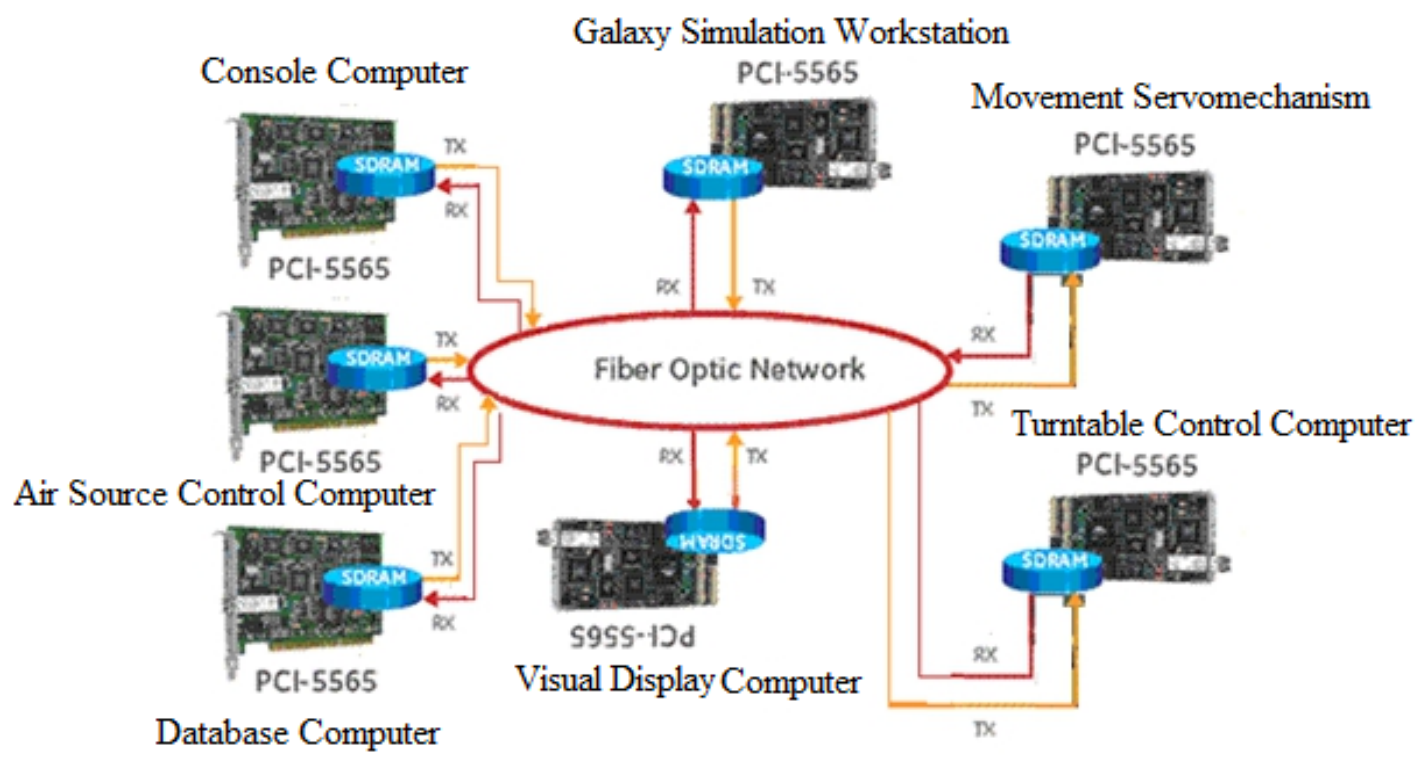

Fig. 2: The reflective memory network framework for the semi-physical simulation of the laser beam rider guidance missile

During the simulation process, the console computer sends the kind information of the participating objects, the initial state information between the missile and target, the starting signal and the normal state/abort signal of the test and the interference and noise data to the Galaxy Simulation Workstation. The main functions of the Galaxy Simulation Workstation are to compute 
missile models, to obtain the control instruction of the environment simulation facilities, to read the data of the sensors and to control the simulation process. The database computer is used to record the simulation data and support the data for the console computer. The visual display computer is responsible for real-time displaying the movement state of the missile. The functions of the console computer are to read the motion information of the missile from the Galaxy Simulation Workstation, then, to obtain the control command to control the environmental unit. At the same time, the console computer can also read the state information from the environmental unit and sent them to the Galaxy Simulation Workstation.

When the real-time simulation begins, the Galaxy Simulation Workstation sent the different simulation parameters to the physical simulators every millisecond based on the different simulation stages. At the same time, it sends the commands to the control simulator for the parameters loading work. After the loading of the parameters, every physical simulator sends a real-time physical simulation data to the Galaxy Simulation Workstation. Then, it is sent to the simulation nodes of every computer by the simulation management and control computer.

\subsection{The network connection}

In the network connecting process, the current mainstream reflective memory card of VMIPCI-5565 system is used as the main transmission medium. The memory capacity of this card is 128Mbyte. It also has the 4Kbyte FIFO.

The optical fiber VMICBL-000-F3-102 is used for the data transfer. The interface form is shown in figure 3. The length is 25 foot. The interposition loss rate $\leqslant 0.3 \mathrm{~dB}$. The reflection loss rate $\geqslant 35 \mathrm{~dB}$.

When each node uses the reflective memory card, the reflective memory card should be open firstly [3]:

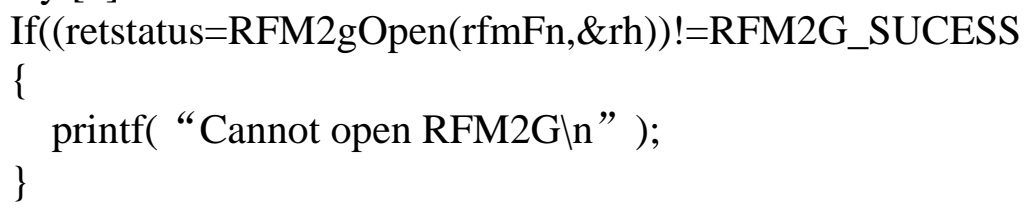

When the simulation is completed, the statement RFM2gClose(\&rh) is used to close the memory card. It makes the reflective memory card stay in the non-operating state.

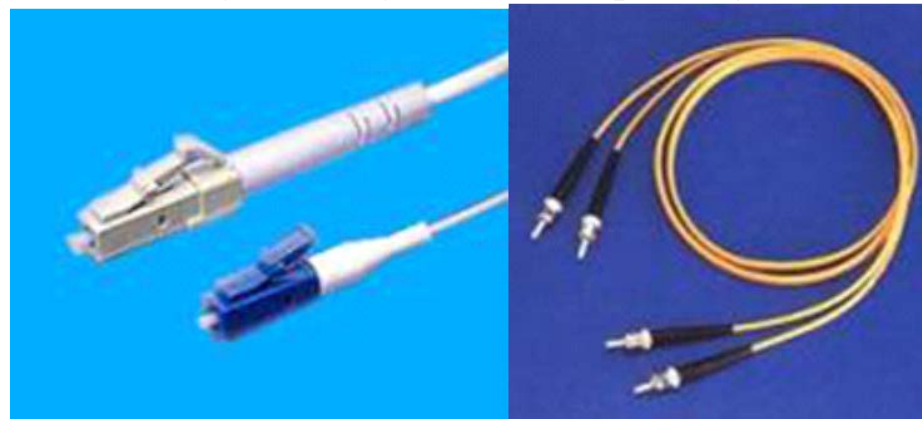

Fig. 3: The interface form

All computer nodes in the simulation should be in the open state. The Galaxy Simulation Workstation firstly receives the initialization data from the console computer, and then controls the simulation process. Equipment control computer read the motion information of the missile from the Galaxy Simulation Workstation. Then it generates the control commands and controls the environmental equipment.

\subsection{Data transmission}

VMIPCI-5565 can provide four network interruptions. Any processor can interrupt on the other network nodes. In addition, by means of the write operation of a register, any processor can also interrupt on all network nodes.

In order to achieve the write operations, VMIPCI - 5565 which is as a source generates a special package, including: a strobe pulse, the ID of the sending node, the ID of the destination node and 32-bit data. The specific implementation is as follows:

retstatus=RFM2gEnableEvent(rh,RFM2GEVENT_INTR1); 
retstatus=RFM2gSendEvent(rh,RFM2GEVENT_INTR1);

retstatus=RFM2gWrite(rh, wAddress, $\left(\right.$ void*$\left.^{*}\right)(\&$ w_d $\left.), n\right)$;

When detects the ID of the destination node in the packet and the strobe pulse accords with itself, the receiving node will send the ID of the node and data which are stored in one of the four FIFO whose depth is 127. These four FIFO respectively correspond to four interrupts. After the information stores in the FIFO, if it gets the permission of the software, the receiving node will sent the interrupt to the local processor. The 32-bit data which are stored in the FIFO are definable for the users. It usually is viewed as the interrupt vector. As a part of the interrupt service, the local processor reads this information from the FIFO, and then makes the corresponding response. The specific implementation is as follows:

retstatus=RFM2gWaitForEvent(rh,RFM2GEVENT_INTR1);

retstatus=RFM2gDisableEvent(rh,RFM2GEVENT_INTR1).

\subsection{Error management of data transmission}

In error management, firstly, through the use of the error detection equipment which has a fibre channel encoding/decoding and additional cyclic redundancy code and check. VMIPCI-5565 card can detect the error. Once the node detects errors, error data will be removed from the system and generate an interrupt [4].

Secondly, the FIFO characteristic of the product data transmission is used to prevent data overflow when the system stay at a high data rate. When the FIFO is about full, a bit in the status register will be setting. This is an indicator for node software system, meaning to hang the operation of write image memory, until the data in the FIFO is less than half of the capacity. Once the FIFO is full, the write operation of image memory will receive a STOP *. And the data will not be lost.

The third is to use redundant transmission mode.VMIPCI-5565 will be configurated in a redundant transmission mode by removing a template jumper. In this mode, no matter the size of the dynamic package, each posted online bag is sent twice. The receiving circuit of each node in the network is to evaluate each redundancy transmission. If no error is detected in the first transmission, the transmission will be used to update the memory on the plate, and a second transmission is discarded. On the contrary, if there is an error in transmission for the first time, use the second transport to update the memory on the plate under the premise of no error in the second transport. It rarely happens when two redundant transmissions are both wrong.

Once happened, two transmissions are not used and the data will be completely deleted from the network. Through the above three aspects of the error management of data transmission, the reliability of data transmission in the driving beam of laser guided missile hardware-in-the-loop simulation of reflective memory network can be effectively improved.

\subsection{Various nodes of network monitoring}

There is a date which can verify whether the data pass the loop in the state register. In other words, the loop is not broken. It also can be used to measure the response time of the network. Through the monitor of this state, it can be a good judgment to judge whether the ring network is closed or open.

At the same time, at the beginning of the simulation, the console computer sends a symbolic data to the specific address of each computer to computer simulation nodes. After the target computer receives the data, it feeds back a signature data to the corresponding address of the computer console section. Thus, it can realize that each point in the loop is fluent in the initial stage of the simulation.

\section{The use of optical fiber reflective memory network real-time simulation and testing}

After the completion of the network, we respectively finish the simulation testing without the hardware load and hardware load simulation testing. 8878 times point-to-point transmission test results without the hardware load are shown in Fig.4. 


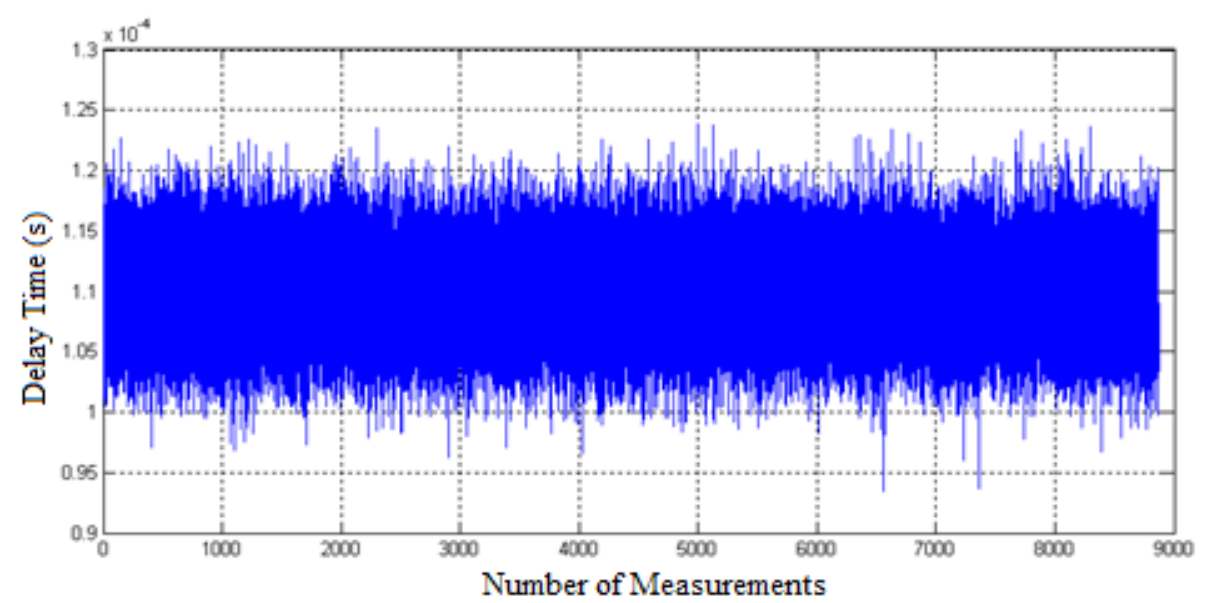

Fig. 4: Point-to-point transmission network latency for zero load condition

From the figure 4We can see that the average of the single point-to-point network transmission delay is 0.11 millisecond, and the maximum is 0.125 millisecond. The reflective memory network of the semi-physical simulation of the laser beam rider guidance missile has 7 nodes. So the biggest delay cannot more than 0.8 millisecond. Because during the simulation, some node computer need not to feedback data, the simulation requirements of $1 \mathrm{~ms}$ can be satisfied adequately.

The simulation objects are introduced into the network one by one. After the simulation test, the largest network delay is not greater than $1 \mathrm{~ms}$. The time test results of one full load simulation is shown in Fig. 5.

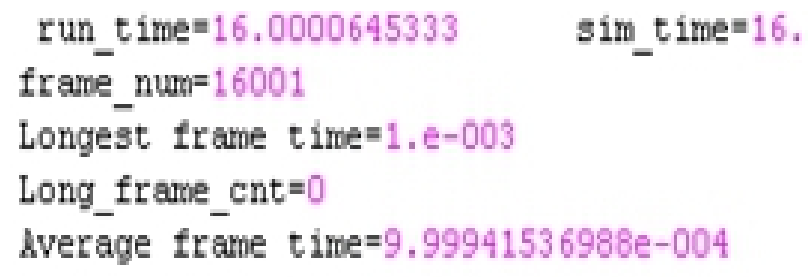

speed_up=0.999995966682

Fig. 5: Time test results of one semi-physical simulation of the laser beam rider guidance missile

In multiple semi-physical simulation experiments, it proves that the communication of the network system is stable and the data transmission error rate is low.No matter in the case of no-load and load, it can run steady.

\section{Summary}

According to the needs of the system, this paper focuses on the network communication which is a key factor of the semi-physical simulation. Because the optical fiber reflective memory network is introduced in the semi-physical simulation of the laser beam rider guidance missile, the communication speed of the network significantly increased. At the same time, because this data communication system is limited in a small range, the standard protocol is not necessary to be applied in the communication protocol. The direct data transmission is used in the process. There is no spending of the software system in the transmission process. By the memory mapping mechanism of the operating system, it can implement the data transmission directly skipping the driver.

\section{References}

[1] Wang Henglin, Cao Jianguo: The design and application of the simulation system. Beijing: Science Press (2003).

[2] Liu Zaozhen, Wei Hualiang: System simulation. Beijing: Beijing Institute of Technology Press, 2004. 
[3] Hu Shangxu: Digital simulation of Distributed systems Dynamics. The information and control. (1983), p. 57-63

[4] Common REM2g Application Program Interface(API) and Command Line Inter Preter for VMISFT/RFM2G Driver. USA.2004.

[5] Liao Ying, Liang JiaHong: Real-time simulation theory and supporting technology. Changsha: National University ofDefense Technology Press (2002). 